\title{
Caracterización clínica y microbiológica de la bacteriemia por Staphylococcus aureus
}

\author{
Clinical and microbiological characterization of \\ bacteremia by Staphylococcus aureus
}

\author{
Carolina Hincapié-Osorno, César Caraballo-Cordovez, \\ María Fernanda Tibaduiza-García, Daniela de Jesús Garcés-Rodríguez, \\ Lina Echeverri-Toro, Johana Ascuntar-Tello, Alba Luz León-Álvarez, \\ Fabián Jaimes-BarRagán • MedelLín (Colombia)
}

\section{Resumen}

Introducción: Staphylococcus aureus es una frecuente causa de bacteriemias y el incremento en las tasas de resistencia dificulta su tratamiento inicial. La incidencia de bacteriemias por S. aureus resistente a meticilina (SAMR) ha aumentado, principalmente en las infecciones adquiridas en el medio hospitalario. Este estudio pretende caracterizar clínicamente a los pacientes con bacteriemia por $S$. aureus detectados en un hospital de Medellín y definir su perfil de resistencia microbiana.

Métodos: cohorte retrospectiva en un hospital de tercer nivel. Se incluyeron pacientes $\geq 16$ años con aislamiento para Staphylococcus aureus en sangre periférica. Se registró información relacionada con el episodio, el sitio de adquisición, la clasificación microbiológica y las características clínicas.

Resultados: de 775 reportes de hemocultivos positivos para $S$. aureus, finalmente se ingresaron 698 pacientes al estudio. La mediana de edad fue de 58 años (RIQ=42-69), 383 eran hombres (54.9\%). En 354 pacientes (50.7\%) la bacteriemia era de origen nosocomial y el $26.2 \%(n=183)$ eran SAMR. En 123 (17.6\%) bacteriemias adquiridas en la comunidad, la frecuencia de SAMR fue $33.3 \%(n=41)$. El antecedente más frecuente fue tener un catéter vascular en 321 pacientes $(46 \%)$. Se inició terapia empírica en $67.2 \%$ de los casos $(n=469)$. Respecto a la gravedad, la mediana del puntaje SOFA fue de 4 (RIQ=1-5), APACHE II de 15 (RIQ=10-19) y la mortalidad fue del $24.9 \%(n=174)$.

Conclusiones: a pesar de que la mayoría de bacteriemias son de origen nosocomial, el reporte más frecuente fue SAMS tanto en el grupo nosocomial como en el de la comunidad. Sin embargo, la mortalidad en el grupo de bacteriemia nosocomial fue inferior comparada con la de la comunidad. (Acta Med Colomb 2018; 43: 200-206).

Palabras clave: Staphylococcus aureus, bacteriemia, mortalidad, prescripción antibiótica.

\footnotetext{
Abstract

Introduction: Staphylococcus aureus is a frequent cause of bacteremia and the increase in resistance rates makes initial treatment difficult. The incidence of bacteraemia due to methicillinresistant S. aureus (MRSA) has increased, mainly in infections acquired in the hospital environment. This study aims to clinically characterize patients with $S$. aureus bacteremia detected in a Medellín hospital and to define their microbial resistance profile.

Methods: retrospective cohort in a tertiary hospital. Patients $\geq 16$ years with isolation for Staphylococcus aureus in peripheral blood were included. Information related to the episode, the acquisition site, the microbiological classification and the clinical characteristics were recorded.

Results: Of 775 reports of blood cultures positive for S. aureus, 698 patients were finally admitted to the study. The median age was 58 years (RIQ = 42-69), 383 were men $(54.9 \%)$. In 354 patients $(50.7 \%)$ the bacteremia was of nosocomial origin and $26.2 \%(n=183)$ were MRSA. In 123 (17.6\%) bacteremia acquired in the community, the frequency of MRSA was $33.3 \%(n=41)$. The most frequent antecedent was to have a vascular catheter in 321 patients (46\%). Empirical therapy was initiated in $67.2 \%$ of the cases $(n=469)$. Regarding severity, the SOFA score median was 4 $(\mathrm{RIQ}=1-5)$, APACHE II of $15(\mathrm{RIQ}=10-19)$ and mortality was $24.9 \%(\mathrm{n}=174)$.
}

Dres. Carolina Hincapié-Osorno, César Caraballo-Cordovez, María Fernanda TibaduizaGarcía, Daniela de Jesús Garcés-Rodríguez: Médicos y Cirujanos Universidad de Antioquia. Grupo Académico de Epidemiología Clínica (GRAEPIC); Dra. Lina Echeverri-Toro: Médica Microbióloga Fundación Hospitalaria San Vicente de Paúl; Johana Ascuntar-Tello y Alba Luz León-Álvarez: Grupo Académico de Epidemiología Clínica (GRAEPIC). Gerentes de Sistemas de Información en Salud, Universidad de Antioquia; Dr. Fabián Jaimes-Barragán: Especialista en Medicina Interna. Grupo Académico de Epidemiología Clínica (GRAEPIC). Profesor Departamento de Medicina Interna Universidad de Antioquia. Director de Investigaciones Fundación Hospitalaria San Vicente de Paúl. Medellín (Colombia).

Correspondencia: Dr. Fabián Jaimes. Medellín (Colombia).

E-mail: fabian.jaimes@udea.edu.co

Recibido: 13/VIII/2017Aceptado: 1/X/2018 
Conclusions: although the majority of bacteremia are of nosocomial origin, the most frequent report was MSSA in both the nosocomial and community groups. Despite this, mortality in the nosocomial bacteremia group was lower compared with that of the community. (Acta Med Colomb 2018; 43: 200-206).

Key words: Staphylococcus aureus, bacteremia, mortality, antibiotic prescription.

\section{Introducción}

Staphylococcus aureus es el patógeno bacteriano más comúnmente aislado en humanos (1), con un espectro infeccioso bastante amplio desde infecciones cutáneas superficiales hasta condiciones amenazantes como bacteriemia, endocarditis, neumonía, síndrome de choque tóxico y sepsis (2). Está asociado con mayor mortalidad que cualquier otro microorganismo (3) y es la principal causa de bacteriemias en numerosas áreas geográficas (3). $S$. aureus infecta frecuentemente en el ámbito hospitalario y en la comunidad y es un problema creciente que genera aumento tanto en los costos del sistema de salud, como en morbilidad y mortalidad. El incremento en las tasas de resistencia dificulta el enfoque inicial de la bacteriemia por S. aureus (4). Desde los últimos años la incidencia de bacteriemias por $S$. aureus resistente a meticilina (SAMR) ha aumentado, principalmente en las infecciones adquiridas en el medio hospitalario (5). No obstante, la bacteriemia por $S$. aureus sensible a meticilina (SAMS) continúa siendo un problema importante (4).

La epidemiología de las cepas de $S$. aureus y su resistencia es muy cambiante según el área geográfica e incluso entre diferentes hospitales (6). El curso de la infección es diferente dependiendo de la resistencia y el lugar de adquisición del microorganismo y se ha sugerido que la infección por SAMR tiene una mayor mortalidad asociada que la infección debida a SAMS (7). La instauración de una terapia empírica inapropiada o retardada se encuentra relacionada con el aumento de la mortalidad a corto plazo y con los días de estancia hospitalaria (8). Sin embargo, el tratamiento de la bacteriemia debe ser encaminado idealmente según el patrón de resistencia del germen, ya que la evidencia actual demuestra que la vancomicina, que ha sido usada durante muchos años como terapia empírica, tiene resultados subóptimos en el tratamiento de infecciones por SAMS (4).

En Latinoamérica y específicamente en Colombia el estudio de $S$. aureus ha estado más enfocado en la descripción del SAMR (2). Con respecto a la bacteriemia por $S$. aureus en Colombia existen estudios publicados sobre factores de mortalidad en pacientes con cáncer y sobre mortalidad asociada específicamente a la bacteriemia por $\operatorname{SAMR}(9,10)$. El propósito de este estudio fue caracterizar clínicamente a los pacientes con bacteriemia por S. aureus detectados en un hospital de tercer nivel de atención de la ciudad de Medellín y definir su perfil de resistencia microbiana.

\section{Material y Métodos \\ Diseño, lugar y participantes}

Se realizó un estudio de cohorte retrospectivo en el Hospital Universitario San Vicente de Paúl, Medellín, Colombia. El hospital es un centro de tercer nivel de atención, de carácter universitario, con 672 camas de las cuales 470 son de hospitalización de adultos distribuidas de la siguiente manera: 45 camas de cuidados intensivos, 30 de cuidados intermedios y 395 en salas generales.

Se incluyeron pacientes mayores de 16 años con aislamiento microbiológico en muestras de sangre periférica para Staphylococcus aureus entre enero de 2012 y agosto de 2016. Se excluyeron segundos episodios de un mismo paciente, muestras de catéteres vasculares o de otros tipo pero sin aislamiento en sangre periférica, aquellos sin información disponible en la historia clínica y las bacteriemias polimicrobianas (dos o más gérmenes).

\section{Definición de variables}

Comorbilidades: se evaluó el número y la gravedad de las condiciones asociadas de acuerdo con la clasificación de comorbilidades del índice de Charlson (11), el cual estima la supervivencia a 10 años a partir de la presencia de insuficiencia cardiaca congestiva, enfermedad hepática leve, demencia, hemiplejía o paraplejia, enfermedad renal, tumor primario incluyendo leucemia y linfoma, enfermedad pulmonar crónica, diabetes con complicaciones crónicas, sida/VIH, enfermedad reumatológica, tumor sólido metastásico o enfermedad hepática moderada-grave. Además de las anteriores, se registraron los antecedentes de drogadicción y alcoholismo, uso de esteroides, trasplante de órgano, embarazo, orden de no reanimación y antecedente de endocarditis.

Origen de la bacteriemia: se catalogó según la clasificación de Friedman et al (12) como nosocomial en aquellos pacientes hospitalizados por 48 o más horas; asociada al cuidado de la salud definida como hemocultivo positivo dentro de las primeras 48 horas del ingreso con alguno de los siguientes criterios: terapia intravenosa domiciliaria en los últimos 30 días, cuidado de heridas, hemodiálisis, quimioterapia intravenosa, hospitalización igual o mayor a dos días en los últimos 90 días o residencia en un lugar con asistencia médica; y la bacteriemia adquirida en la comunidad como hemocultivo positivo dentro las primeras 48 horas de hospitalización en pacientes quienes no cumplían criterios para bacteriemia asociada al cuidado de la salud.

Clasificación microbiológica: la muestra de los hemocultivos se procesó en el equipo Bact-alert (bioMérieux ${ }^{\circledR}$ ), 
una vez marcó un cultivo como positivo, se realizó coloración de Gram y posteriormente se sembró en el medio de cultivo sólido correspondiente (Agar MacConkey). Una vez se obtuvo crecimiento de colonias bacterianas, éstas fueron llevadas al equipo VITEKTWO (bioMérieux ${ }^{\circledR}$ ) para hacer la identificación del género y especie del microorganismo, además de la realización del antibiograma que permitió conocer la presencia o no de resistencia a antibióticos, teniendo en cuenta las concentraciones inhibitorias mínimas (MIC, por sus siglas en inglés) determinadas por el Instituto de Estándares Clínicos y de Laboratorio de Estados Unidos del 2012 (CLSI, por sus siglas en inglés).

Resistencia a la meticilina: según $C L S I$, los criterios de interpretación para oxacilina en Staphylococcus aureus son, si MIC $\leq 2$ se considera sensible, si MIC $\geq 4$ se considera resistente.

\section{Otras variables relacionadas}

Se documentó la presencia en los pacientes de prótesis valvulares, prótesis ortopédicas, ventilación mecánica previa al cultivo; catéteres intravasculares centrales, procedimientos quirúrgicos, trauma o quemaduras en los últimos tres meses. Prescripción de antibióticos (nombre, dosificación, duración); gravedad de la enfermedad valorada usando la puntuación APACHE II (Acute Physiologic and Chronic Health Evaluation II) (13) y la frecuencia y magnitud de la disfunción de órganos se midió con la puntuación SOFA (14), estas puntuaciones se determinaron con la información obtenida del registro.

Se clasificó la gravedad de la enfermedad como sepsis grave como aquella disfunción orgánica aguda secundaria a infección y choque séptico como la sepsis grave sumada a hipotensión no revertida con reanimación mediante fluidos; de acuerdo con la guía de supervivencia en sepsis (15).

Se reportó el número de horas en que el Bact-alert se demoró en reportar microorganismos en el hemocultivo inicial; de la misma forma se hizo con el hemocultivo de control.

\section{Variables de desenlace}

Muerte en la misma hospitalización del ingreso al estudio, tiempo de estancia hospitalaria, necesidad de ingreso a unidad de cuidados intensivos posterior al cultivo.

\section{Fuente de la información}

La información clínica fue recolectada de forma retrospectiva únicamente a partir de la historia clínica y de las notas de enfermería. Se recolectó la información relacionada con la toma de la muestra del hemocultivo, los signos vitales y los exámenes de laboratorios más próximos a la toma de la muestra. Todo el seguimiento y registro de información se realizó por parte del personal capacitado para la recolección de los datos.

\section{Tamaño de muestra}

Dado que el diseño del estudio es de tipo descriptivo retrospectivo en aquellos pacientes con estafilococcemia presentada entre 2012 y 2015, no se hizo un cálculo de tamaño de muestra.

\section{Plan de análisis}

De las variables cualitativas se obtuvieron frecuencias absolutas, frecuencias relativas y proporciones; y las variables cuantitativas se expresaron de acuerdo con su distribución en promedio y su respectiva desviación estándar o en mediana y su respectivo rango intercuartílico.

\section{Resultados}

De 1467 reportes de hemocultivos positivos para $S$. aureus, luego de exclusiones, se tuvieron como elegibles a 761 pacientes (Figura 1).

Las características clínicas y demográficas de la población del estudio se presentan en la Tabla 1. En 354 pacientes $(50.7 \%)$ la bacteriemia era de origen nosocomial y $26.2 \%$ del total $(n=183)$ eran SAMR. El factor asociado con la bacteriemia con mayor frecuencia fue tener al menos un catéter venoso central o ambulatorio en 321 pacientes (46\%). La mortalidad total fue del $24.9 \%$ (174/698).

Se inició terapia empírica en el $67.2 \%$ de los casos $(n=469)$, en la Tabla 2 se muestra en detalle la terapia empírica administrada, dividida en seis grupos según el origen de la bacteriemia (nosocomial, intermedia, de la comunidad) y su resistencia (SAMR y SAMS).

La mortalidad más alta se encontró en el grupo de SAMS adquirido en la comunidad en $40.2 \%$ (33/82) y en este mismo grupo se encontró la necesidad de UCI posterior al hemocultivo más alto: 36.6\% (30/82) (Tabla 3).

\section{Discusión}

En esta cohorte restrospectiva de pacientes con bacteriemia por $S$. aureus en un hospital universitario, se encontró

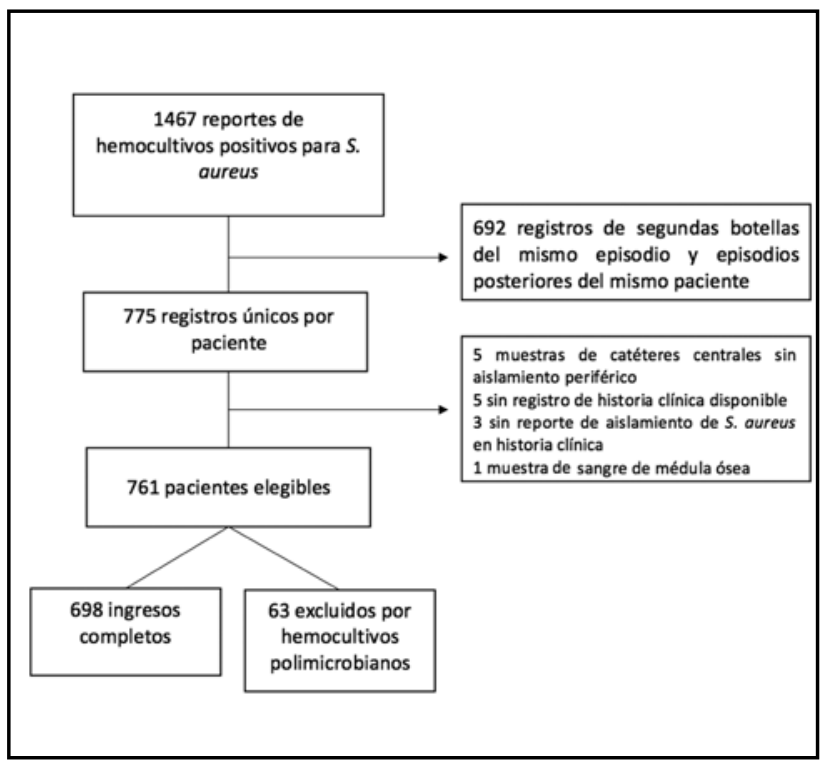

Figura 1. Flujograma de captación de pacientes según resultado de hemocultivos. 
Tabla 1. Características generales y evolución de los participantes del estudio.

\begin{tabular}{|c|c|}
\hline Características de la población & $(n=698)$ \\
\hline Edad (RIQ) & $58 \quad(42-69)$ \\
\hline Género masculino & $383(54.9 \%)$ \\
\hline \multicolumn{2}{|c|}{ Factores relacionados al momento del hemocultivo } \\
\hline Prótesis valvulares & $14(2.0 \%)$ \\
\hline Prótesis ortopédica & $10(1.4 \%)$ \\
\hline Ventilación Mecánica & $69(9.9 \%)$ \\
\hline Catéter IV centrales y/o ambulatorios & $321(46 \%)$ \\
\hline Terapia de remplazo renal & $300(43 \%)$ \\
\hline \multicolumn{2}{|l|}{ En los tres meses previos } \\
\hline Uso de terapia antibiótica & $143(20.5 \%)$ \\
\hline Uso terapia inmunosupresora & $130(18.6 \%)$ \\
\hline Cirugía & $94 \quad(13.5 \%)$ \\
\hline Procedimiento invasivo ambulatorio & $105(15.0 \%)$ \\
\hline Trauma & $157(22.5 \%)$ \\
\hline Quemaduras & $18 \quad(2.6 \%)$ \\
\hline \multicolumn{2}{|l|}{ Causa de ingreso } \\
\hline Infección/ sospecha de infección & $341(48.9 \%)$ \\
\hline Enfermedad general/intoxicación & $314(45 \%)$ \\
\hline Trauma/ quemadura/ Cirugía & $43(6.2 \%)$ \\
\hline \multicolumn{2}{|l|}{ Presunto origen de la bacteriemia } \\
\hline Bacteriemia nosocomial & $354(50.8 \%)$ \\
\hline Bacteriemia asociada al cuidado de la salud & $221(31.6 \%)$ \\
\hline Bacteriemia adquirida en la comunidad & $123(17.6 \%)$ \\
\hline \multicolumn{2}{|l|}{ Sospecha foco primario } \\
\hline Desconocido & $105(15.0 \%)$ \\
\hline Piel y tejidos blandos/ISO sup-prof & $189 \quad(27.1 \%)$ \\
\hline Línea venosa periférica CVP/FAV & $82 \quad(11.8 \%)$ \\
\hline Línea central CVC/CAC/PICC & $273 \quad(39.1 \%)$ \\
\hline Pulmonar/ foco respiratorio & $34 \quad(4.9 \%)$ \\
\hline Otro & $15 \quad(2.2 \%)$ \\
\hline Puntaje Charlson (RIQ) & $2(1-3)$ \\
\hline Horas positivo inicial (RIQ) & $12.6(10.7-15.5)(n=650)$ \\
\hline Horas positivo control (RIQ) & $16.9(13.7-23.4)(n=107)$ \\
\hline SAMR & $183 \quad 26.2 \%$ \\
\hline \multicolumn{2}{|l|}{ MIC vancomicina } \\
\hline MIC $<=0.5$ & $247 \quad 35.4 \%$ \\
\hline MIC 1 & $442 \quad 63.3 \%$ \\
\hline MIC 2 & $4 \quad 0.6 \%$ \\
\hline Sin dato & $0.7 \%$ \\
\hline \multicolumn{2}{|l|}{ Gravedad del cuadro } \\
\hline SOFA (RIQ) & $4(1-6)$ \\
\hline APACHE II (RIQ) & $15(10-19)$ \\
\hline \multicolumn{2}{|c|}{$\begin{array}{l}\text { Abreviaciones: ISO, infección sitio operatorio; CVP, catéter venoso periférico; FAV, fistula } \\
\text { arterio venosa; CVC, catéter venoso central; CAC, catéter arterial central; PICC, catéter central } \\
\text { de inserción periférica; MIC, concentración mínima inhibitoria; SOFA, Sepsis-related Organ } \\
\text { Failure Assessment; APACHE II, Acute Physiology And Chronic Health Evaluation; SAMR } \\
\text { Staphylococcus aureus metelicino-resistente. }\end{array}$} \\
\hline
\end{tabular}

que más de $50 \%$ de las mismas eran de origen nosocomial y el SAMS fue el patógeno más prevalente, aislado en $73.8 \%$ del total de las muestras. Lo anterior difiere de lo reportado actualmente en países desarrollados, donde el SAMR supera el 50\% (16, 17). En Estados Unidos, Wisplinghoff y colaboradores mostraron que la proporción de SAMR se incrementó de 22\% en 1995 a 57\% en 2001 (18). Todo esto sugiere que el comportamiento del SAMR en nuestro medio se asemeja al reportado en décadas pasadas en países industrializados y, como hemos encontrado en otras investigaciones (19), nuestra ecología bacteriana parece seguir con aproximadamente 20 años de retraso, lo que ocurre en las naciones más industrializadas.

Con respecto a la proporción de bateriemias por $S$. aureus adquiridas en la comunidad, Mylotte y colaboradores reportaron en 1987 que la estafilococcemia proveniente de la comunidad era $14 \%$ contra $86 \%$ proveniente del hospital (20), aclarando que para este entonces no se diferenciaban estas infecciones realmente nosocomiales con respecto a las simplemente asociadas al cuidado de la salud. Friedman y colaboradores en el año 2002 reportaron para las estafilococcemias un porcentaje de $14.5 \%$ para las adquiridas en la comunidad, $46 \%$ asociadas al cuidado de la salud y $39 \%$ a las nosocomiales (12). En nuestro estudio encontramos una distribución similar para la comunidad (17.6\%), pero diferente para las asociadas al cuidado de la salud y las nosocomiales, con $31.6 \%$ y $50.8 \%$, respectivamente. A pesar de lo anterior, la prevalencia de SAMR en cada uno de los subgrupos difiere significativamente entre nuestros resultados y los del estudio de Friedman: mientras nosotros hallamos SAMR en $26.8 \%$ de los aislamientos nosocomiales, $21.3 \%$ de los asociados al cuidado de la salud y $33.3 \%$ en la comunidad, ellos describieron la proporción de SAMR de 61,52 y $14 \%$, respectivamente (12). Un estudio realizado en Argentina entre el 2002 y 2013 por Togneri y colaboradores, reportó una prevalencia del SAMR de $37 \%$ del total de aislamientos de la comunidad, similar al reportado en nuestro estudio. Sin embargo, el SAMR del total asociado a la asistencia sanitaria se encontró en 63\% (21), lo cual es superior a nuestros hallazgos pero se asemeja más a resultados de otros estudios como el de Friedman y Mylote $(12,20)$.

Kaasch y colaboradores realizaron un análisis de 3395 pacientes con bacteriemia por $S$. aureus, incluidos en cinco estudios prospectivos realizados entre 2006 y 2011 en 20 centros médicos de tercer nivel en Alemania, España, Reino Unido y Estados Unidos (22). La mortalidad general fue de $40.7 \%$, superior a $24.9 \%$ que reportamos en esta cohorte, aunque la frecuencia de infección por MRSA $(20.6 \%)$ y la fuente en catéter venoso periférico o central (27.7\%), fueron similares a las encontradas en nuestro estudio. Es de resaltar que la mortalidad en nuestra cohorte fue mayor en el grupo con infección por SAMS proveniente de la comunidad. Con respecto a la mortalidad por patrones de resistencia exclusivamente, se describen diferencias tan amplias como en el estudio de Chang y colaboradores, donde los pacientes 
Tabla 2. Terapia antibiótica empírica según fuente de la infección y tipo de resistencia.

\begin{tabular}{|c|c|c|c|c|c|c|c|}
\hline & \multicolumn{2}{|c|}{ Nosocomial $(n=354)$} & \multicolumn{2}{|c|}{$\begin{array}{l}\text { Asociada al cuidado de la salud } \\
\qquad(\mathrm{n}=\mathbf{2 2 1})\end{array}$} & \multicolumn{2}{|c|}{ Comunidad $(\mathrm{n}=123)$} & \multirow{2}{*}{$\begin{array}{c}\begin{array}{c}\text { Todos } \\
(\mathrm{n}=698) \\
(\%)\end{array}\end{array}$} \\
\hline & $\begin{array}{c}\text { SAMS } \\
(n=259) \\
(73.2 \%)\end{array}$ & $\begin{array}{c}\text { SAMR } \\
(n=95) \\
(26.8 \%)\end{array}$ & $\begin{array}{c}\text { SAMS } \\
(n=174) \\
(78.7 \%)\end{array}$ & $\begin{array}{c}\text { SAMR } \\
(\mathrm{n}=47) \\
(21.3 \%)\end{array}$ & $\begin{array}{c}\text { SAMS } \\
(n=82) \\
(66.7 \%)\end{array}$ & $\begin{array}{c}\text { SAMR } \\
(n=41) \\
(33.3 \%)\end{array}$ & \\
\hline Oxacilina & $1(0.4)$ & 0 & 0 & 0 & 0 & 0 & $1(0.1)$ \\
\hline Betalactamicos & $50(19.3)$ & $19(20.0)$ & $24(13.8)$ & $9(19.2)$ & $18(21.9)$ & $8(19.5)$ & $129(18.5)$ \\
\hline Glicopeptidos & 30 (11.6) & $10(10.5)$ & $19(10.9)$ & $5(10.6)$ & $6(7.3)$ & $4(9.8)$ & $74(10.6)$ \\
\hline Otros & $8(3.1)$ & $2(2.1)$ & $3(1.7)$ & 0 & $1(1.2)$ & $1(2.4)$ & $15(2.2)$ \\
\hline Betalactamicos+ glicopeptidos & $73(28.2)$ & $23(24.2)$ & $79(45.4)$ & $19(40.4)$ & $20(24.4)$ & $11(26.8)$ & $225(32.2)$ \\
\hline Betalactamicos + glicopeptidos + otros & $3(1.2)$ & 0 & $2(1.2)$ & 0 & $3(3.7)$ & $2(4.9)$ & $10(1.4)$ \\
\hline Betalactamicos + otros & $2(0.8)$ & $2(2.1)$ & $1(0.6)$ & 0 & $4(4.9)$ & $2(4.9)$ & $11(1.6)$ \\
\hline Glicopeptidos + otros & $1(0.4)$ & $2(2.1)$ & $2(1.2)$ & 0 & 0 & 0 & $5(0.7)$ \\
\hline Sin terapia empírica & $91(35.1)$ & $37(38.9)$ & $44(25.3)$ & $14(29.8)$ & $30(36.6)$ & $13(31.7)$ & $229(32.8)$ \\
\hline
\end{tabular}

Tabla 3. Descenlaces de acuerdo con la fuente de infección y el tipo de resistencia

\begin{tabular}{|c|c|c|c|c|c|c|c|}
\hline & \multicolumn{2}{|c|}{ Nosocomial $(n=354)$} & \multicolumn{2}{|c|}{$\begin{array}{l}\text { Asociada al cuidado de la salud } \\
\qquad(\mathrm{n}=221)\end{array}$} & \multicolumn{2}{|c|}{ Comunidad (n=123) } & \multirow{2}{*}{$\begin{array}{c}\text { Total } \\
(\mathbf{n}=698) \\
(\%)\end{array}$} \\
\hline & $\begin{array}{c}\text { SAMS } \\
(n=259) \\
(73.2 \%)\end{array}$ & $\begin{array}{c}\text { SAMR } \\
(n=95) \\
(26.8 \%)\end{array}$ & $\begin{array}{c}\text { SAMS } \\
(n=174) \\
(78.7 \%)\end{array}$ & $\begin{array}{c}\text { SAMR } \\
(n=47) \\
(21.3 \%)\end{array}$ & $\begin{array}{c}\text { SAMS } \\
(\mathrm{n}=82) \\
(66.7 \%)\end{array}$ & $\begin{array}{c}\text { SAMR } \\
(n=41) \\
(33.3 \%)\end{array}$ & \\
\hline Vegetación ecocardio & $\begin{array}{l}14(10.8) \\
(\mathrm{n}=130)\end{array}$ & $\begin{array}{l}2(4.4) \\
(\mathrm{n}=45)\end{array}$ & $\begin{array}{l}14(12.7) \\
(\mathrm{n}=110)\end{array}$ & $\begin{array}{l}1(3.2) \\
(\mathrm{n}=31)\end{array}$ & $\begin{array}{l}3(7.9) \\
(\mathrm{n}=38)\end{array}$ & $\begin{array}{c}1(4) \\
(\mathrm{n}=25)\end{array}$ & $\begin{array}{l}36(9.5) \\
(\mathrm{n}=380)\end{array}$ \\
\hline Siembras & $\begin{array}{l}57(22.1) \\
(\mathrm{n}=258)\end{array}$ & $\begin{array}{c}20(21.1) \\
(\mathrm{n}=95)\end{array}$ & $\begin{array}{l}25(14.4) \\
(\mathrm{n}=174)\end{array}$ & $\begin{array}{c}9(19.2) \\
(\mathrm{n}=47)\end{array}$ & $\begin{array}{c}24(29.6) \\
(\mathrm{n}=81)\end{array}$ & $\begin{array}{c}13(31.7) \\
(\mathrm{n}=41)\end{array}$ & $\begin{array}{c}149(21.4) \\
(\mathrm{n}=697)\end{array}$ \\
\hline Sepsis grave & $\begin{array}{c}147(57.7) \\
(\mathrm{n}=255)\end{array}$ & $\begin{array}{c}54(56.8) \\
(\mathrm{n}=95)\end{array}$ & $\begin{array}{c}85(49.4) \\
(n=172)\end{array}$ & $\begin{array}{c}22(47.8) \\
(n=46)\end{array}$ & $\begin{array}{c}53(65.4) \\
(n=81)\end{array}$ & $\begin{array}{c}26(63.4) \\
(n=41)\end{array}$ & $\begin{array}{c}388(56.2) \\
(\mathrm{n}=691)\end{array}$ \\
\hline Choque séptico & $\begin{array}{l}51(20) \\
(n=255)\end{array}$ & $\begin{array}{c}17(17.9) \\
(\mathrm{n}=95)\end{array}$ & $\begin{array}{c}22(12.79) \\
(n=172)\end{array}$ & $\begin{array}{c}6(13.0) \\
(n=46)\end{array}$ & $\begin{array}{c}22(27.2) \\
(\mathrm{n}=81)\end{array}$ & $\begin{array}{c}9(21.9) \\
(n=41)\end{array}$ & $\begin{array}{c}127(18.4) \\
(n=691)\end{array}$ \\
\hline UCI posterior hemocultivo & $63(24.3)$ & $21(21.1)$ & $34(19.5)$ & $8(17.0)$ & $30(36.6)$ & $11(26.8)$ & $167(23.9)$ \\
\hline Muerte al alta & $66(25.5)$ & $25(26.3)$ & $27(15.5)$ & $11(23.4)$ & $33(40.2)$ & $12(29.3)$ & $174(24.9)$ \\
\hline
\end{tabular}

con bacteriemia por SAMR exhibieron una mortalidad de $50 \%$, comparada con $23 \%$ en los pacientes con SAMS (23). De igual manera, en un estudio retrospectivo realizado por Kobayashi y colaboradores en Japón con 340 pacientes con bacteriemia por $S$. aureus (24), la mortalidad global fue de $34.7 \%$, la frecuencia de infección por MRSA de $44.4 \%$ y la mortalidad asociada a este grupo fue de $39.7 \%$, comparada con $30.7 \%$ en el grupo infectado por SAMS. Este mismo estudio reportó un origen de la bacteriemia en la comunidad en $27 \%$ de los casos, valor cercano al porcentaje encontrado en nuestro estudio. Respecto a la mortalidad según el sitio de infección; autores como Kaech y colaboradores en un estudio retrospectivo en un hospital suizo, describen que de 308 episodios de bacteriemia por S. aureus, $26 \%$ de los provenientes de la comunidad fallecieron, a diferencia de los de origen nosocomial en que dicho desenlace se presentó en $13 \%$ (25). Nuestro estudio también mostró mayor mortalidad en los pacientes con infecciones de la comunidad, pero con proporciones mayores a las encontradas en el estudio suizo.

Varios estudios de cohorte han reportado menores efectos en desenlaces clínicos, o eficacia disminuida, en bacteriemias por SAMS tratadas con vancomicina $(26,27)$, por lo que la bacteremia por SAMS debería ser siempre tratada de primera elección con penicilinas antiestafilocócicas o cefalosporinas de primera generación. A pesar de esta recomendación, en nuestra cohorte se empleó en la terapia empírica oxacilina solamente en un paciente y apenas en $20 \%$ se inició un betalactámico. Casi la mitad de los 
pacientes recibieron empíricamente glicopéptidos solos o en combinación, y en una tercera parte no se consideró la necesidad de un tratamiento empírico. Aunque no es posible establecer y valorar los criterios clínicos con base en los cuales los médicos decidieron iniciar terapia empíríca y escogieron el antibiótico de elección, la relativamente baja prevalencia de SAMR de nuestra cohorte (26.2\%) refuerza la necesidad de la educación continua en la prescripción adecuada de antibióticos.

En nuestro medio encontramos sólo un estudio con características similares al que realizamos (2), específicamente con respecto a la mortalidad en bacteriemia por $S$ aureus discriminada tanto por el patrón de resistencia (SAMR vs SAMS) como por el lugar de adquisición (comunidad vs institución). A pesar de incluir un número similar de pacientes $(n=810)$, los años de reclutamiento fueron diferentes (2008-2010) y las distribuciones por grupo difieren con las nuestras: bacteriemia nosocomial $40.9 \%(n=331)$, bacteriemia asociada al cuidado de la salud $46.8 \%(n=379)$ y bacteriemia adquirida en la comunidad $12.3 \%(n=100)$. La mortalidad fue más frecuente entre las infecciones nosocomiales MRSA (12.8\%) y las asociadas al cuidado de la salud MSSA $(8.8 \%)$.

Nuestro trabajo tiene la ventaja de un número de pacientes significativamente mayor, comparado con otros estudios de este tipo realizados en nuestro medio. Como limitaciones, por tratarse de un estudio retrospectivo tiene mayor riesgo de obtener información inexacta o incompleta para algunos episodios de bacteriemia. Así mismo, por ser realizado en un solo centro de alta complejidad y de referencia para la región, tiene limitaciones en la posibilidad de generalizar sus resultados. A pesar de que en Colombia la prevalencia de cepas resistentes es baja comparada con otros países, el manejo de la bacteriemia por Staphylococcus aureus es un problema mundial cuyo tratamiento es motivo de amplio debate. Por esta razón, consideramos que estudios posteriores con mayor tamaño de muestra deben evaluar la magnitud de los desenlaces clínicos discriminados de acuerdo con el patrón de resistencia y el lugar de adquisición, además de considerar la pertinencia y eficacia de las medidas usadas en su tratamiento. Adicionalmente, se deben realizar estudios que busquen determinar con mayor exactitud el riesgo y la presentación clínica de la bacteriemia por $S$. aureus de origen de la comunidad, ya que su sospecha temprana y manejo oportuno podrían disminuir sus complicaciones y mortalidad.

\section{Conclusiones}

En una cohorte de pacientes con bacteriemia por $S$. aureus en un hospital universitario de referencia en una ciudad capital, más del $80 \%$ de las mismas fueron de origen nosocomial o asociadas al cuidado de la salud; sin embargo, el aislamiento más frecuente fue el SAMS en todos los subgrupos. La mayor mortalidad se observó en los pacientes con bacteriemia adquirida en la comunidad y particularmente en aquellos infectados con SAMS.

\section{Financiación}

Trabajo apoyado parcialmente por la Estrategia de Sostenibilidad de la Universidad de Antioquia, 2013-2014.

\section{Referencias}

1. David MZ, Daum RS. Community-associated methicillin-resistant Staphylococcus aureus: epidemiology and clinical consequences of an emerging epidemic. Clin Microbiol Rev. 2010;23:616-87. https://doi.org//10.1128/CMR.00081-09.

2. Jimenez JN, Ocampo AM, Vanegas JM, Rodriguez EA, Mediavilla JR, Chen $\mathbf{L}$, et al. A comparison of methicillin-resistant and methicillin-susceptible Staphylococcus aureus reveals no clinical and epidemiological but molecular differences. Int J Med Microbiol. 2013;303:76-83. https://doi.org//10.1016/j.jimm.2012.12.003.

3. Naber CK, Baddour LM, Giamarellos-Bourboulis EJ, Gould IM, Herrmann M, Hoen B, et al. Clinical consensus conference: survey on Gram-positive bloodstream infections with a focus on Staphylococcus aureus. Clin Infect Dis. 2009;48:S260-70. https://doi.org//10.1086/598185.

4. Shorr AF, Kunkel MJ, Kollef M. Linezolid versus vancomycin for Staphylococcus aureus bacteraemia: pooled analysis of randomized studies. J Antimicrob Chemother. 2005;56:923-9.

5. Paul M, Kariv G, Goldberg E, Raskin M, Shaked H, Hazzan R, et al. Importance of appropriate empirical antibiotic therapy for methicillin-resistant Staphylococcus aureus bacteraemia. J Antimicrob Chemother. 2010;65:2658-65. https://doi.org//10.1093/jac/dkq373.

6. Naves KS, Vaz da Trindade N, Gontijo Filho PP. Methicillin-resistant Staphylococcus aureus bloodstream infection: risk factors and clinical outcome in nonintensive-care units. Rev Soc Bras Med Trop. 2012;45:189-93.

7. Rehm SJ, Tice A. Staphylococcus aureus: methicillin-susceptible S. aureus to methicillin-resistant S. aureus and vancomycin-resistant S. aureus. Clin Infect Dis. 2010;51:S176-82. https://doi.org//10.1086/653518.

8. Lodise TP, McKinnon PS, Swiderski L, Rybak MJ. Outcomes analysis of delayed antibiotic treatment for hospital-acquired Staphylococcus aureus bacteremia. Clin Infect Dis. 2003;36:1418-23.

9. Tibavizco D, Rodriguez JY, Silva E, Cuervo SI, Cortes JA. Therapeutic approach to Staphylococcus aureus bacteremia. Biomedica. 2007;27:294-307.

10. Cuervo SI, Cortes JA, Sanchez R, Rodriguez JY, Silva E, Tibavizco D, et al. Risk factors for mortality caused by Staphylococcus aureus bacteremia in cancer patients. Enferm Infecc Microbiol Clin. 2010;28:349-54. https://doi.org//10.1016/j. eimc.2009.06.015.

11. Charlson ME, Pompei P, Ales KL, MacKenzie CR. A new method of classifying prognostic comorbidity in longitudinal studies: development and validation. Journal of chronic diseases. 1987;40:373-83.

12. Friedman ND, Kaye KS, Stout JE, McGarry SA, Trivette SL, Briggs JP, et al. Health care--associated bloodstream infections in adults: a reason to change the accepted definition of community-acquired infections. Ann Intern Med. 2002;137:791-7.

13. Knaus WA, Draper EA, Wagner DP, Zimmerman JE. APACHE II: a severity of disease classification system. Crit Care Med. 1985;13:818-29.

14. Vincent JL, Moreno R, Takala J, Willatts S, De Mendonca A, Bruining H, et al. The SOFA (Sepsis-related Organ Failure Assessment) score to describe organ dysfunction/failure. On behalf of the Working Group on Sepsis-Related Problems of the European Society of Intensive Care Medicine. Intensive Care Med. 1996;22:707-10.

15. Rhodes A, Evans LE, Alhazzani W, Levy MM, Antonelli M, Ferrer R, et al. Surviving Sepsis Campaign: International Guidelines for Management of Sepsis and Septic Shock: 2016. Intensive Care Med. 2017;43:304-77. https://doi. org//10.1007/s00134-017-4683-6.

16. Rhee Y, Aroutcheva A, Hota B, Weinstein RA, Popovich KJ. Evolving Epidemiology of Staphylococcus aureus Bacteremia. Infect Control Hosp Epidemiol. 2015;36:1417-22. https://doi.org//10.1017/ice.2015.213.

17. Bassetti M, Peghin M, Trecarichi EM, Carnelutti A, Righi E, Del Giacomo P, et al. Characteristics of Staphylococcus aureus Bacteraemia and Predictors of Early and Late Mortality. PLoS One. 2017;12:e0170236. https://doi.org//10.1371/ journal.pone. 0170236 .

18. Wisplinghoff H, Bischoff T, Tallent SM, Seifert H, Wenzel RP, Edmond MB. Nosocomial bloodstream infections in US hospitals: analysis of 24,179 cases from a prospective nationwide surveillance study. Clin Infect Dis. 2004;39:309-17.

19. Brun-Buisson C, Meshaka P, Pinton P, Vallet B. EPISEPSIS: a reappraisal of the epidemiology and outcome of severe sepsis in French intensive care units. Intensive Care Med. 2004;30:580-8.

20. Mylotte JM, McDermott C, Spooner JA. Prospective study of 114 consecutive episodes of Staphylococcus aureus bacteremia. Rev Infect Dis. 1987;9:891-907.

21. Togneri AM, Podesta LB, Perez MP, Santiso GM. Study of Staphylococcus 
aureus infections in a general acute care hospital (2002-2013). Rev Argent Microbiol. 2017;49:24-31. https://doi.org//10.1016/j.ram.2016.09.006.

22. Kaasch AJ, Barlow G, Edgeworth JD, Fowler VG, Jr., Hellmich M, Hopkins S, et al. Staphylococcus aureus bloodstream infection: a pooled analysis of five prospective, observational studies. $J$ Infect. 2014;68:242-51. https://doi. org//10.1016/j.jinf.2013.10.015.

23. Chang FY, MacDonald BB, Peacock JE, Jr., Musher DM, Triplett P, Mylotte JM, et al. A prospective multicenter study of Staphylococcus aureus bacteremia: incidence of endocarditis, risk factors for mortality, and clinical impact of methicillin resistance. Medicine (Baltimore). 2003;82:322-32.

24. Kobayashi D, Yokota K, Takahashi O, Arioka H, Fukui T. A predictive rule for mortality of inpatients with Staphylococcus aureus bacteraemia: A classifica- tion and regression tree analysis. Eur J Intern Med. 2014;25:914-8. https://doi. org//10.1016/j.ejim.2014.10.003.

25. Kaech C, Elzi L, Sendi P, Frei R, Laifer G, Bassetti S, et al. Course and outcome of Staphylococcus aureus bacteraemia: a retrospective analysis of 308 episodes in a Swiss tertiary-care centre. Clin Microbiol Infect. 2006;12:345-52.

26. Kim SH, Kim KH, Kim HB, Kim NJ, Kim EC, Oh MD, et al. Outcome of vancomycin treatment in patients with methicillin-susceptible Staphylococcus aureus bacteremia. Antimicrob Agents Chemother. 2008; 52: $192-7$.

27. Stryjewski ME, Szczech LA, Benjamin DK, Jr., Inrig JK, Kanafani ZA, Engemann JJ, et al. Use of vancomycin or first-generation cephalosporins for the treatment of hemodialysis-dependent patients with methicillin-susceptible Staphylococcus aureus bacteremia. Clin Infect Dis. 2007; 44: 190-6. 